\title{
Tratamento cirúrgico das dissecções de aorta tipo A utilizando parada cardiocirculatória total com hipotermia profunda
}

Luciano Cabral ALBUQUERQUE*, Marco Antônio GOLDANI*, Juremir Joāo GOLDANI*, Ricardo Medeiros PIANTÁ*, Rubens Lorentz ARAÚJO*, Joāo Batista PETRACCO*

RBCCV 44205-194

ALBUQUeRQUE, L. C.; GOLDANI, M. A.; GOLDANI, J. J.; PIANTÁ, R. M.; ARAÚJO, R. L.; PETRACCO, J. B. - Tratamento cirúrgico das dissecçōes de aorta tipo A utilizando parada cardiocirculatória total com hipotermia profunda. Rev. Bras. Cir. Cardiovasc., 8(1):1-8, 1993.

RESUMO: No período de julho de 1986 a julho de 1993, 22 pacientes portadores de dissecção de aorta tipo A foram tratados cirurgicamente utilizando-se parada cardiocirculatória (PCC) total sob hipotermia profunda $\left(18^{\circ} \mathrm{C}\right)$, dos quais 15 apresentavam dissecçāo aguda e 7 dissecçāo crônica. Em 14 casos $(64 \%)$ a aorta ascendente foi reconstruída utilizando-se enxerto reto de Dacron, com troca valvar aórtica em 5 pacientes e ressuspensáo valvar aórtica em 2; a reconstrução do arco aórtico foi empregada em 8 casos (36\%), nos quais a dissecção se extendia ou se originava no mesmo, havendo necessidade de reimplante dos ramos supra-aórticos em 3 pacientes. O tempo médio do $\mathrm{PCC}$ foi de 43 minutos, a mortalidade hospitalar foi de $18 \%$, e a complicação pós-operatória mais freqüente foi a infecçāo respiratória. Cinco pacientes $(22,7 \%)$ apresentaram dano neurológico pós-operatório, sendo, em 4 casos, reversível e atribuível a edema cerebral; 1 caso $(4,5 \%)$ apresentou acidente vascular hemisférico estabelecido. Concluímos que a técnica de PCC sob hipotermia profunda deve ser utilizada sempre no reparo das dissecçōes de aorta tipo $A$, independentemente de sua extensão ao arco aórtico, pois oferece uma proteção cerebral segura, permite a inspeção ampla da zona dissecada e do local de rotura da íntima, e evita o trauma produzido pelo clampeamento da aorta acometida.

DESCRITORES: aorta, dissecçōes, cirurgia; parada circulatória; hipotermia profunda.

\section{INTRODUÇĀO}

A dissecção aórtica é, reconhecidamente, a mais grave lesão que acomete a aorta. Desde a classificação estabelecida por De BAKEY et alii ${ }^{8}$, em 1965, muito se avançou no conhecimento de sua fisiopatogenia e de seu comportamento biológico.

Já em 1970, a constatação de que as dissecçôes que comprometiam a aorta ascendente apresentavam um comportamento mais agressivo e um curso clínico menos favorável, devendo, por isso, serem diferenciadas para uma abordagem terapêutica mais precoce e também mais agressiva, levou
DAILY et alii ${ }^{7}$ a proporem modificação na classificação inicial, criando a classificação de Stanford, mais comumente utilizada nos dias atuais, a qual agrupa todos os casos que envolvem a aorta ascendente em dissecçōes do tipo A e designa, como dissecçōes do tipo $B$, aquelas com comprometimento exclusivo da aorta descendente (Figura 1).

No tratamento das dissecçōes do tipo A, o aprimoramento e a sistematização nas técnicas de diagnóstico, monitorização e suporte hemodinâmico per-operatórios, técnicas anestésicas e, principalmente, nos métodos de reconstrução cirúrgica e de proteção cerebral, ocorridos nos últimos anos, têm 


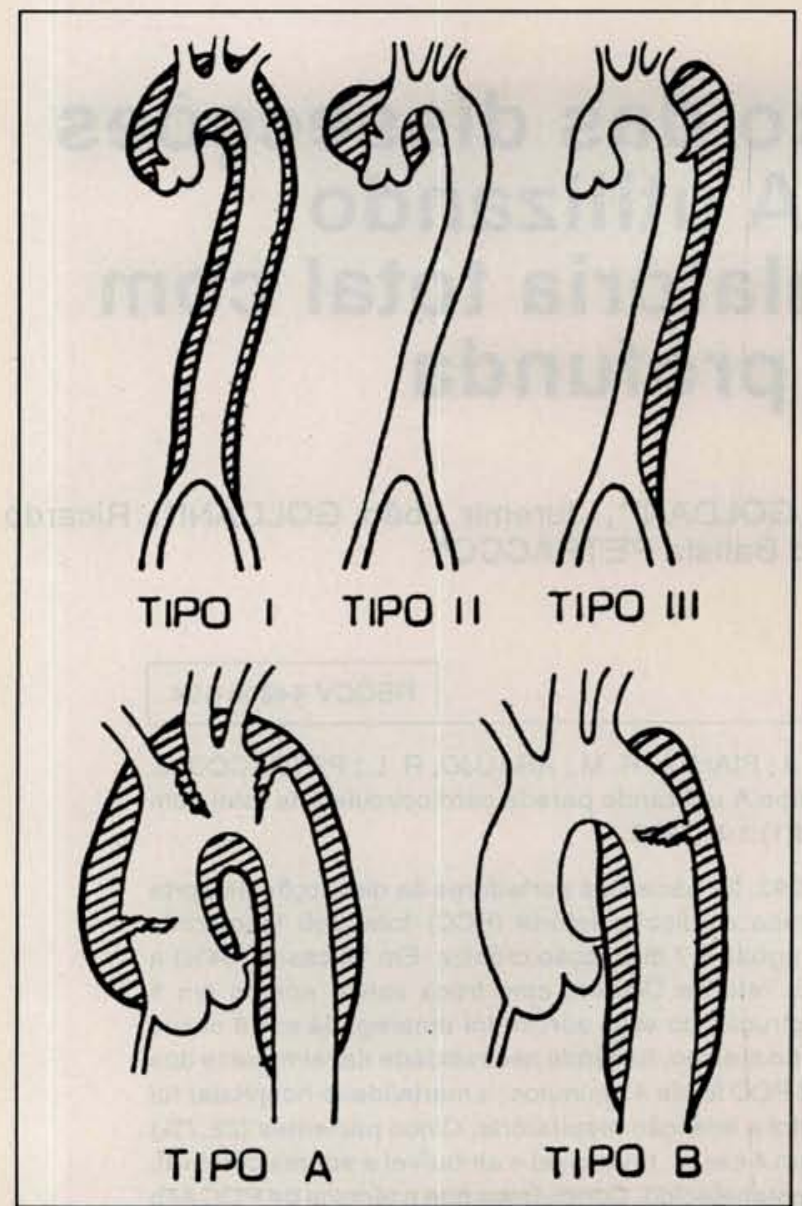

Fig. 1 - Acima, a classificação clássica de De Bakey em 3 tipos: abaixo, a classificaçăo mais recente de Stanford, onde o tipo A engloba todos os casos de dissecçáo com envolvimento da aorta ascendente.

contribuído para índices de morbi-mortalidade progressivamente melhores.

Entretanto, alguns aspectos permanecem sendo motivo de controvérsia. De modo particular, o estabelecimento de um método seguro de proteção cerebral durante o reparo das dissecçōes do tipo A e, sobretudo, se este método está indicado somente nos casos com envolvimento do arco transverso, ou se deve ser utilizado como técnica rotineira, tem motivado constante questionamento.

Atualmente, vários grupos têm relatado sucesso utilizando as mais variadas técnicas, como parada cardiocirculatória total (PCC) sob hipotermia profunda, PCC hipotérmica com perfusão seletiva cerebral, normo ou hipotérmica, e PCC hipotérmica com perfusāo cerebral retrógrada pela veia cava superior, entre outras.

Igualmente, os critérios de inclusão do arco aórtico no reparo cirúrgico não se encontram uni- formemente estabelecidos, fazendo com que a decisāo de reconstruir o arco transverso e, principalmente, realizá-lo de forma parcial ou total, seja conflituosa a muitos cirurgiōes.

$\mathrm{Na}$ verdade, independentemente da técnica utilizada, $O$ adequado manejo cirúrgico das dissecçōes do tipo A deve, ao seu final, atender aos seguintes requisitos: 1) remover o sítio da laceraçāo intimal; 2) redirecionar o fluxo à luz verdadeira; 3 ) restaurar a competência valvar aórtica, quando a insuficiência estiver presente; 4) corrigir a isquemia coronária, se necessário, e 5) manter a integridade de perfusão dos ramos supra-aórticos.

A exemplo de alguns grupos, temos utilizado rotineiramente a técnica de $\mathrm{PCC}$ total sob hipotermia de $18^{\circ} \mathrm{C}$ durante a reconstrução cirúrgica de todos os casos de dissecção do tipo $A$, com ou sem extensão aórtico, e descrever esta experiência de 7 anos, apresentando seus resultados imediatos e comparando-os com os relatos de outros autores, - que constitui o objetivo deste estudo.

\section{CASUÍSTICA E MÉTODOS}

Foram analisados, retrospectivamente, os prontuários de 22 pacientes portadores de dissecção aórtica do tipo $A$, submetidos a correçāo cirúrgica no período de julho de 1986 a julho de 1993. Destes, $13(59 \%)$ eram do sexo masculio e $(41 \%)$ do sexo feminino. A idade variou de 33 a 74 anos, havendo maior predominância $(59 \%)$ na faixa dos 40 aos 60 anos; a idade média dos pacientes foi de 53,2 anos.

Todos os pacientes apresentavam dor torácica e $90 \%$ encontravam-se hipertensos na admissão. Em 7 casos $(31,8 \%)$ havia regurgitaçāo valvar aórtica e, em 5 destes, sinais clínicos de insuficiência cardíaca esquerda; derrame pericárdico estava presente em 5 casos $(22,7 \%)$ e 2 pacientes $(9 \%)$ apresentavam síndrome de Marfan.

Quinze pacientes tinham sintomas há menos de 15 dias (dissecção aguda) e, em 7 casos, a cirurgia foi realizada com mais de 15 dias de evolução da doença (dissecção crônica). Todos foram submetidos a exame angiográfico para diagnóstico; 10 pacientes $(45,4 \%)$ realizaram, também, estudo hemodinâmico, por suspeitar-se de isquemia coronária associada, ou previamente à aortografia, pela suspeita inicial de angina ou infarto do miocárdio; em 7 casos $(31,8 \%)$ a tomografia computadorizada foi utilizada como método associado, tendo havido 1 caso de falso negativo nesse grupo. $O$ ecocardiograma trans-esofágico biplanar foi realizado em 2 casos, nos quais havia dúvida arteriográfica, e confirmou o diagnóstico. 
ALBUQUERQUE, L. C.; GOLDANI, M. A.; GOLDANI, J. J.; PIANTÁ, R. M.; ARAÚJO, R. L.; PETRACCO, J. B. - Tratamento cirúrgico das dissecçōes de aorta tipo A utilizando parada cardiocirculatória total com hipotermia profunda. Rev. Bras. Cir. Cardiovasc., $8(1): 1-8,1993$.

A abordagem empregada em todos os casos foi a esternotomia mediana; após heparinização sistêmica $(4 \mathrm{mg} / \mathrm{kg})$, foi procedida a canulaçāo do átrio direito e da artéria femoral e instituída a circulação extracorpórea (CEC), com resfriamento lento a $18^{\circ} \mathrm{C}$ (entre 20 e 30 minutos), à aferição por termômetro nasofaríngeo. Nesse momento, a linha arterial foi clampeada, mantendo-se a drenagem venosa para exangüinaçāo moderada; a CEC foi, então, descontinuada, a PCC estabelecida, a aorta ascendente incisada longitudinalmente e uma sessāo de cardioplegia cristalóide hipotérmica realizada anterogradamente, por canulaçāo direta dos óstios coronários. Durante o período de $\mathrm{PCC}$, foi utilizado resfriamento de superfície com o emprego de gelo tópico na cabeça e pescoço.

A aorta ascendente foi cuidadosamente inspecionada e o estado do arco aórtico avaliado. Em 14 pacientes $(63,6 \%)$, a dissecção encontrava-se restrita à aorta ascendente e a correçăo foi procedida com a interposiçāo de enxerto reto de Dacron e sutura contínua de Polipropileno 3-0, reorientando o fluxo à luz verdadeira. Após realizada a anastomose distal, a CEC foi reinstituída em 8 pacientes, com clampeamento da prótese durante a anastomose proximal; entretanto, em 6 casos nos quais o restabelecimento da CEC com clampeamento da prótese iria restringir o campo operatório, dificultando tecnicamente a execuçāo da anastomose proximal, e nos quais a anastomose distal havia sido realizada num tempo satisfatório e a insuficiência aórtica nāo estava presente, manteve-se a PCC também durante a anastomose proximal.

Em 5 pacientes $(22,7 \%)$ que apresentavam extensão da dissecção ao arco aórtico, foi procedida a reconstruçāo parcial do mesmo ("hemiarch repair"), com enxerto de Dacron e sutura contínua de Polipropileno 3-0, mantendo-se a PCC somente durante a anastomose distal.

Nos 3 casos restantes $(13,6 \%)$, nos quais havia rotura do arco transverso ou o sítio da laceraçāo intimal ali se encontrava, procedeu-se à reconstruçāo total do arco, com enxerto de Dacron e sutura contínua de Polipropileno 3-0, mantendo-se a PCC durante a anastomose distal e o reimplante dos ramos supra-aórticos em bloco (Figura 2).

Em todos os casos, finda a anastomose proximal, o enxerto foi recoberto pela parede nativa da aorta com sutura contínua de Polipropileno 4-0 (endoaneurismorrafia); em alguns casos, foi necessário um esforço com tira circular de Dacron em torno da anastomose distal ("braçadeira") para perfeita hemostasia.

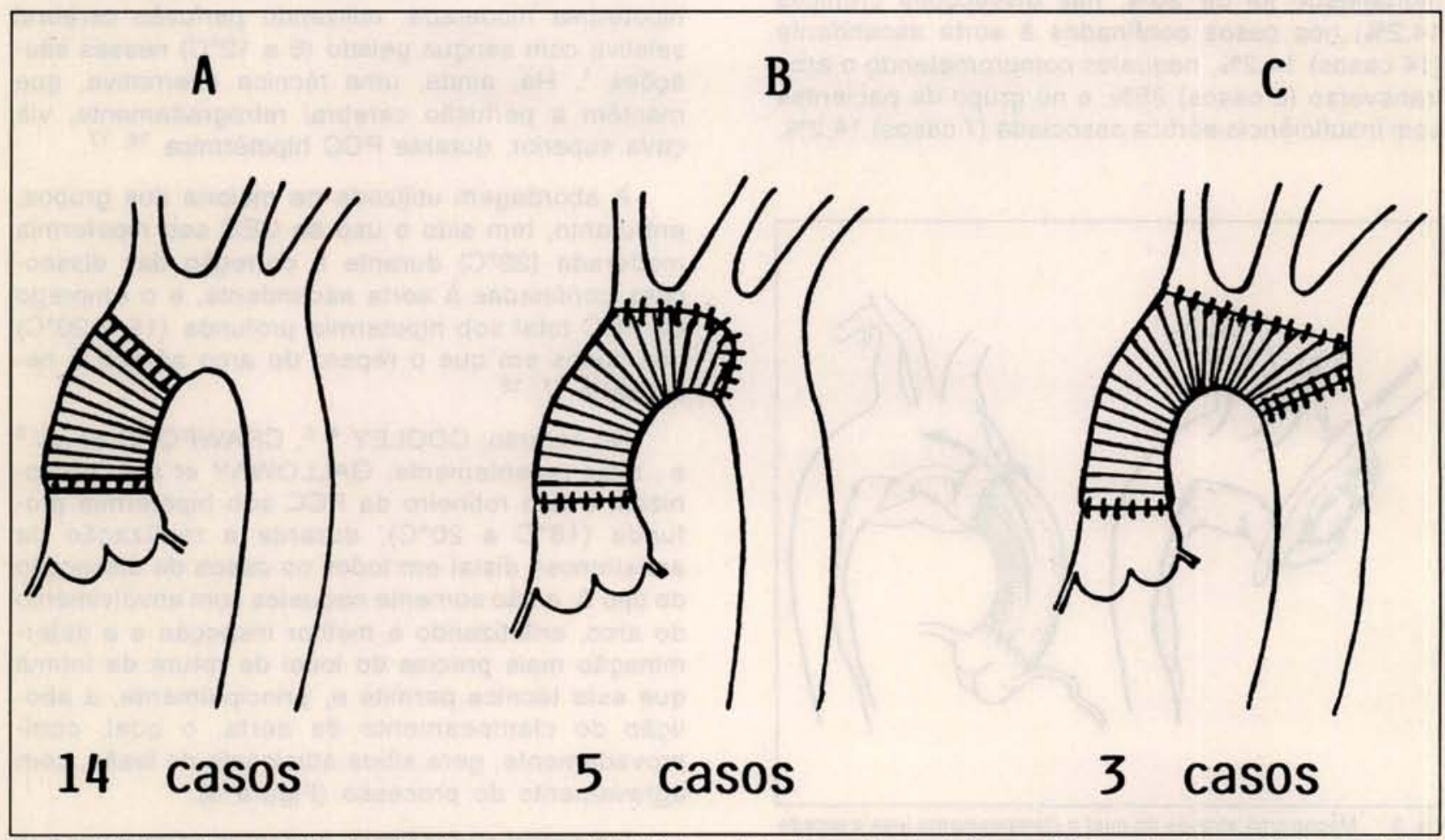

Fig. 2 - Técnicas de reparo cirúrgico utilizadas em nossa série: Al interposição de enxerto reto de Dacron. B) Reconstrução parcial do arco aórtico. C) Reconstrução total do arco aórtico com reimplante dos troncos supra-aórticos. 
ALBUQUERQUE, L. C.; GOLDANI, M. A.; GOLDANI, J. J.; PIANTÁ, R. M.; ARAÚJO, R. L.; PETRACCO, J. B. - Tratamento cirúrgico das dissecçóes de aorta tipo A utilizando parada cardiocirculatória total com hipotermia profunda. Rev. Bras. Cir. Cardiovasc., 8(1):1-8, 1993.

Sete pacientes $(31,8 \%)$ apresentavam insuficiência aórtica: 5 foram submetidos a substituicão valvar por prótese mecânica, devido à rotura de folheto(s) ou à impossibilidade de reconstrução, tendo sido a ressuspensão valvar aórtica possível em apenas 2 casos.

Manobras usuais de retirada de ar foram realizadas durante a reinstituição da $C E C$, o reaquecimento procedido lentamente $\left( \pm 3 \mathrm{~min} / 1^{\circ} \mathrm{C}\right)$ e a CEC descontinuada com temperatura de $37^{\circ} \mathrm{C}$ por no mínimo 5 minutos.

\section{RESULTADOS}

O tempo de parada cardiocirculatória total variou de 22 a 56 minutos (média: $43 \mathrm{~min}$ ), o tempo de isquemia miocárdica entre 40 a 107 minutos (média: $75 \mathrm{~min}$ ) e a duração da circulação extracorpórea oscilou entre 60 e 171 minutos (média: $139 \mathrm{~min}$ ).

A mortalidade hospitalar global da série foi de 18\%. Em 2 casos, o óbito foi trans-operatório, 1 por falência ventricular na saída de perfusão e o outro por sangramento incontrolável. Nos 2 pacientes restantes, o óbito ocorreu no pós-operatório tardio, devido a insuficiência ventilatória $\left(15^{\circ} \mathrm{PO}\right)$ e sépsis $\left(23^{\circ} \mathrm{PO}\right)$, respectivamente.

No grupo de dissecçōes agudas (15 casos), a mortalidade foi de $20 \%$, nas dissecçōes crônicas $14,2 \%$; nos casos confinados à aorta ascendente (14 casos) $14,2 \%$, naqueles comprometendo o arco transverso ( 8 casos) $25 \%$, e no grupo de pacientes com insuficiência aórtica associada ( 7 casos) $14,2 \%$.

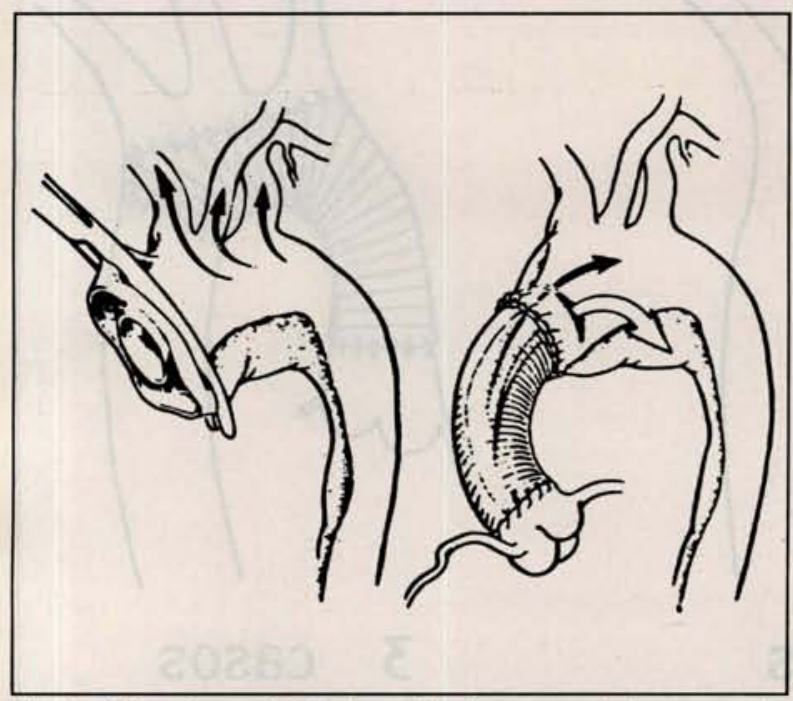

Fig. 3 - Mecanismo através do qual o clampeamento lesa a parede da aorta, criando um novo sítio de laceraçâo distal à reconstrução.
Das complicações pós-operatórias, a infecção respiratória foi a mais freqüente, presente em $36,3 \%$ dos pacientes; arritmias cardíacas ocorreram em $22,5 \%$ dos casos, em sua maioria taquiarritmias supraventriculares transitórias ( 3 casos), e sangramento no pós-imediato com necessidade de reintervenção em $9 \%$.

Houve complicaçōes neurológicas em 5 casos: 4 pacientes $(18 \%)$ apresentaram edema cerebral, traduzido clinicamente por obnubilação prolongada e/ou por dificuldade no desmane ventilatório, comprovados tomograficamente, e remissíveis em, no máximo, 5 dias. Acidente vascular encefálico hemisférico, com dano cerebral permanente, ocorreu em uma paciente $(4,5 \%)$ de 48 anos, cujo tempo de PCC foi de 38 minutos e na qual foi realizada reconstrução parcial do arco aórtico.

\section{COMENTÁRIOS}

O estabelecimento de um método de proteção cerebral seguro durante o reparo das dissecçōes aórticas do tipo A, particularmente as que comprometem o arco transverso, tem representado um permanente desafio e, atualmente, várias técnicas têm sido empregadas. Alguns autores relatam bons resultados utilizando perfusão cerebral seletiva durante período de PCC sob hipotermia profunda, na reconstruçāo do arco aórtico ${ }^{2,15}$; outros propōem a PCC parcial sob hipotermia moderada, utilizando perfusão cerebral seletiva com sangue gelado $\left(6\right.$ a $\left.12^{\circ} \mathrm{C}\right)$ nessas situ-

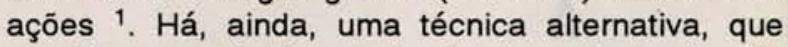
mantém a perfusăo cerebral retrogradamente, via cava superior, durante PCC hipotérmica ${ }^{16,17}$.

A abordagem utilizada na maioria dos grupos, entretanto, tem sido o uso de CEC sob hipotermia moderada $\left(28^{\circ} \mathrm{C}\right)$ durante a correção das dissecçōes confinadas à aorta ascendente, e o emprego da PCC total sob hipotermia profunda $\left(15\right.$ a $\left.20^{\circ} \mathrm{C}\right)$ nos casos em que o reparo do arco aórtico é necessário ${ }^{11}, 12$.

No entanto, COOLEY ${ }^{4,5}$, CRAWFORD et alii ${ }^{6}$ e, mais recentemente, GALLOWAY et alii ${ }^{9}$ preconizam o uso rotineiro da PCC sob hipotermia profunda $\left(18^{\circ} \mathrm{C}\right.$ a $\left.20^{\circ} \mathrm{C}\right)$, durante a realizaçāo da anastomose distal em todos os casos de dissecção do tipo $\mathrm{A}$, e não somente naqueles com envolvimento do arco, enfatizando a melhor inspeção e a determinação mais precisa do local de rotura da íntima que esta técnica permite e, principalmente, a aboliçāo do clampeamento da aorta, o qual, comprovadamente, gera sítios adicionais de lesão, com agravamento do processo (Figura 3 ).

Em nossa experiência, pudemos comprovar que esta técnica realmente facilita a avaliação da exten- 
ALBUQUERQUE, L. C.; GOLDANI, M. A.; GOLDANI, J. J.; PIANTÁ, R. M.; ARAÚJO, R. L.; PETRACCO, J. B. - Tratamento cirúrgico das dissecçōes de aorta tipo A utilizando parada cardiocirculatória total com hipotermia profunda. Rev. Bras. Cir. Cardiovasc., 8(1):1-8, 1993.

são da dissecção e da qualidade das paredes da aorta a nível distal, facilitando, portanto, a tomada de decisão em relação à inclusão do arco aórtico na reconstrução (Figuras 4 e 5 ).

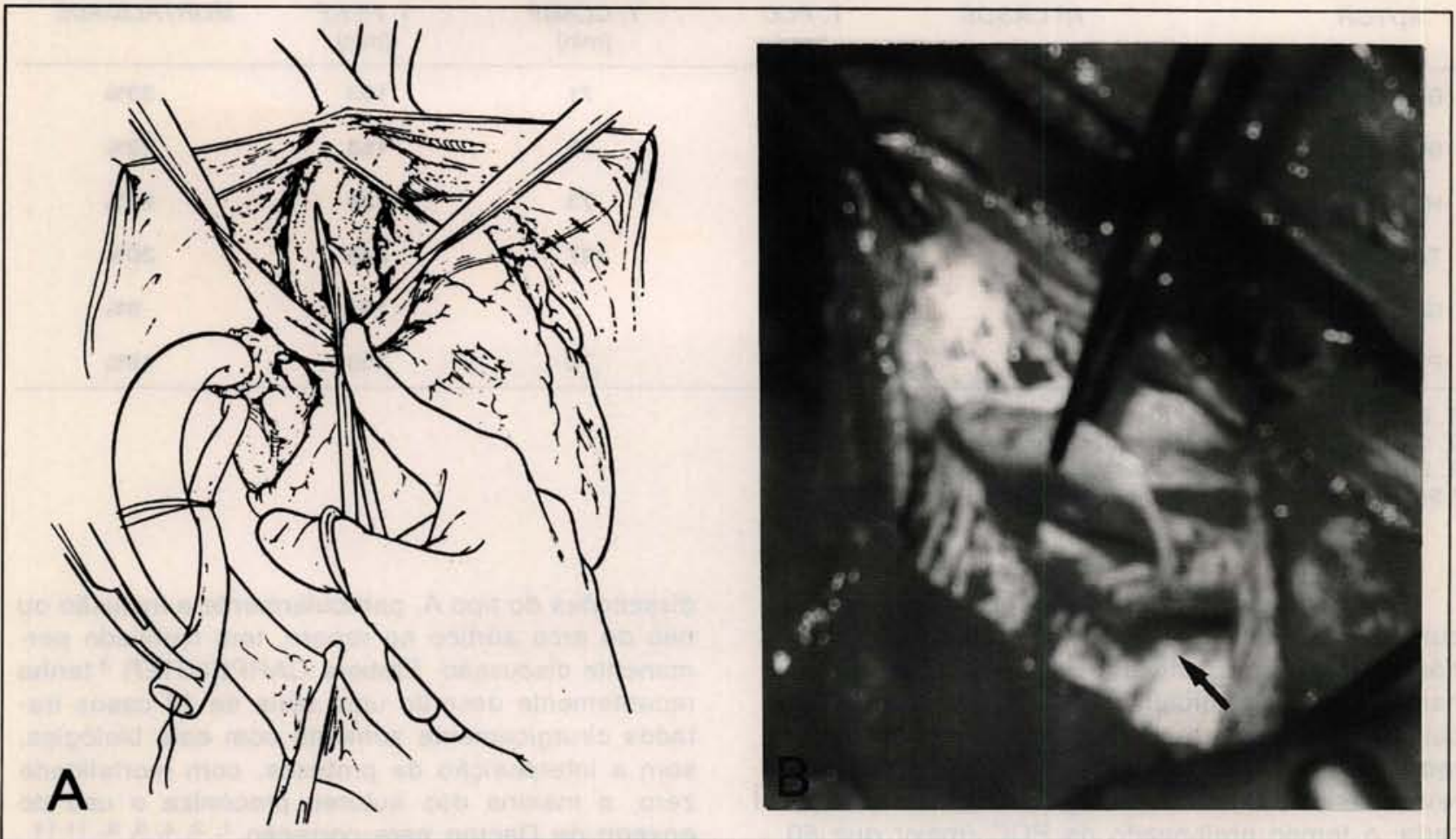

Fig. 4 - A) Abertura longitudinal da aorta ascendente durante PCC total hipotérmica para inspeção da extensão da dissecção e do sítio da laceração intimal. B) Momento trans-operatório correspondente: a seta indica o falso lúmen e o local de rotura da íntima.

A
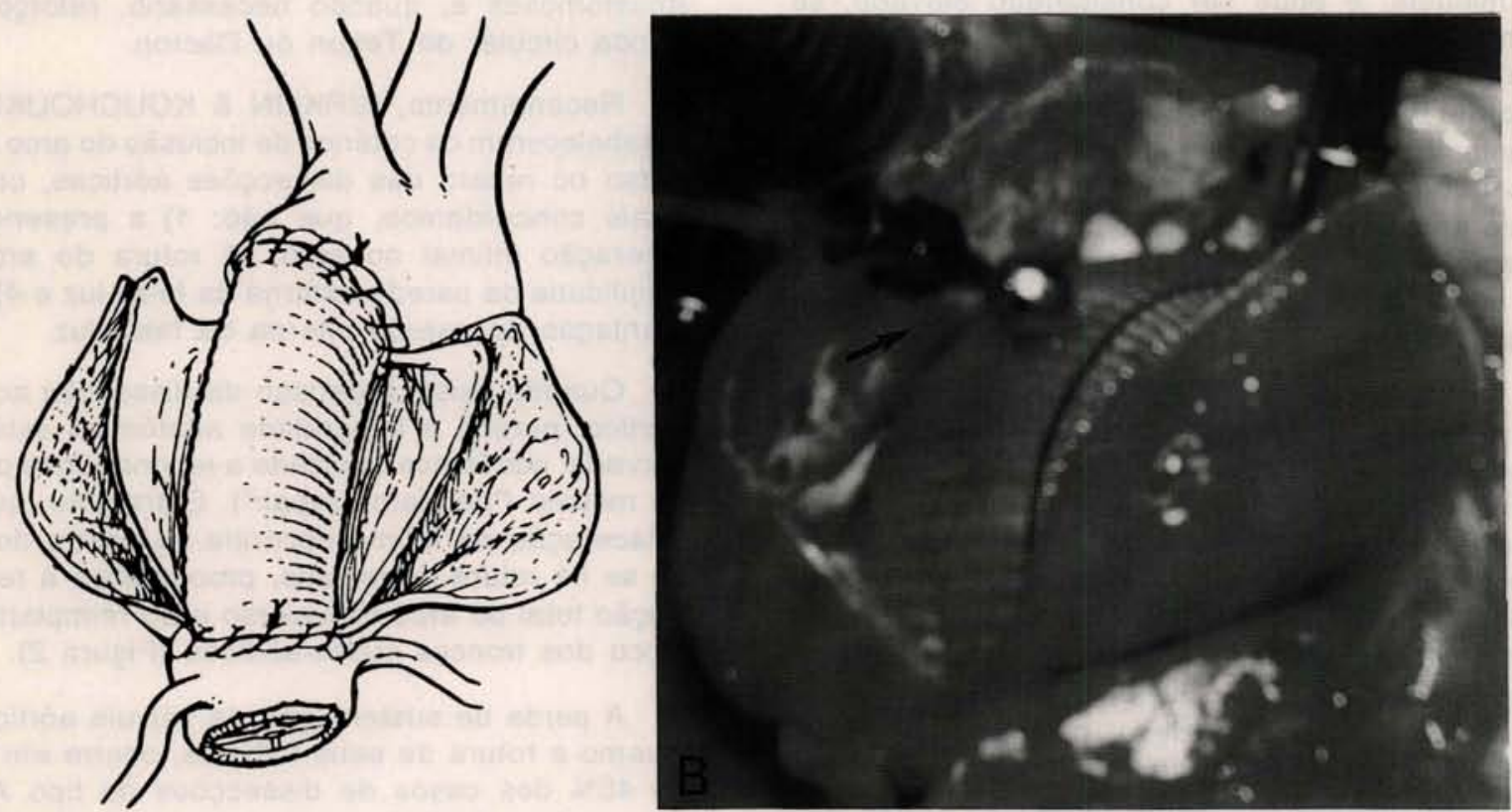

Fig. 5 - A) Interposiçáo de enxerto reto de Dacron na aorta ascendente, o qual será recoberto com a própria parede da aorta (endoaneurismorrafia). B) Representaçáo trans-operatória, ilustrando-se as manobras de retirada de ar. 
ALBUQUERQUE, L. C.; GOLDANI, M. A.; GOLDANI, J. J.; PIANTÁ, R. M.; ARAÚUJ, R. L.; PETRACCO, J. B. - Tratamento cirúrgico das dissecçōes de aorta tipo A utilizando parada cardiocirculatória total com hipotermia profunda. Rev. Bras. Cir. Cardiovasc., $8(1): 1-8,1993$.

TABELA 1

ANÁLISE COMPARATIVA DOS TEMPOS MÉDIOS DE PARADA CARDIOCIRCULATÓRIA, ISQUEMIA MIOCÁRDICA E PERFUSĀO, E DA MORTALIDADE HOSPITALAR DAS SÉRIES RECENTES

\begin{tabular}{|c|c|c|c|c|c|}
\hline AUTOR & $N^{\circ}$ CASOS & $\begin{array}{c}\text { T. } P C C \\
(\min )\end{array}$ & $\begin{array}{l}\text { T. CLAMP } \\
\text { (min) }\end{array}$ & $\begin{array}{l}\text { T. PERF } \\
\text { (min) }\end{array}$ & MORTALIDADE \\
\hline BACHET et alii (1) & *21 & 27 & 71 & 103 & $23 \%$ \\
\hline $\begin{array}{l}(1990) \\
\text { IKONOMIDIS et alii( }{ }^{(12)} \\
\text { (1991) }\end{array}$ & 41 & 25 & 75 & 153 & $32 \%$ \\
\hline $\begin{array}{l}\text { HEINEMANN et alii (11) } \\
\text { (1991) }\end{array}$ & *29 & 24 & 73 & 134 & $20 \%$ \\
\hline $\begin{array}{l}\text { TABAYASHI et alii (15) } \\
\text { (1993) }\end{array}$ & $\star_{20}$ & 32 & 127 & 248 & $30 \%$ \\
\hline GALLOWAY et alii (9) $_{(1993)}$ & 66 & 26 & - & 144 & $9 \%$ \\
\hline PETRACCO et alii & 22 & 43 & 75 & 139 & $18 \%$ \\
\hline
\end{tabular}

T. PCC: Tempo médio de parada cardiocirculatória.

T. Clamp: Tempo médio de isquemia miocárdica.

T. Perf: Tempo médio de circulaçäo extracorpórea.

* Somente dissecçōes com envolvimento do arco aórtico.

Em adição, a melhor compreensão das repercussōes metabólicas da PCC hipotérmica e a experiência relatada por diversos estudos clínicos tornam seu emprego atualmente seguro e apontam os cuidados a serem tomados para evitar dano cerebral. No presente, os principais fatores de risco relacionados a déficit neurológico são a idade avançada, o tempo prolongado de PCC (maior que 60 $\mathrm{min}$ ) e o resfriamento rápido até a hipotermia profunda (menor que $20 \mathrm{~min}$ ) ${ }^{10}$.

Em nossa série, o tempo médio de PCC foi de 43 minutos, e pode ser considerado elevado, se comparado a outros relatos $1,9,11,12,15$ (Tabela 1). Isto se deve ao fato de, diferentemente dos demais autores, termos utilizado a PCC também durante a anastomose proximal em 6 pacientes, nos quais não havia extensão ao arco aórtico nem insuficiência aórtica e a anastomose distal havia sido tecnicamente fácil. Esta medida simplificou a execução da anastomose proximal e nāo adicionou riscos neurológicos.

A incidência de déficit neurológico reversível em nossa série ( $18 \%)$, assim como a ocorrência de dano cerebral permanente $(4,5 \%)$ foi semelhante à observada por outros autores, sendo que o único caso de acidente vascular encefálico não esteve relacionado à idade avançada ( 48 anos) nem à PCC prolongada $38 \mathrm{~min}$ ) 5,10 . De fato, esta observação vem de encontro aos recentes estudos clínicos que sugerem que a incidência de injúria neurológica focal permanente não se deve, na maior parte dos casos, à idade ou tempo de PCC, mas sim a lesōes ateroscleróticas previamente presentes nesse território ${ }^{10}$.

A melhor forma de reconstrução cirúrgica nas dissecçōes do tipo A, particularmente a inclusão ou nāo do arco aórtico no reparo, tem motivado permanente discussão. Embora CARPENTIER ${ }^{3}$ tenha recentemente descrito uma série de 18 casos tratados cirurgicamente somente com cola biológica, sem a interposição de próteses, com mortalidade zero, a maioria dos autores preconiza o uso do enxerto de Dacron para correção $1,2,4,5,9,11-14$.

Dentre as várias técnicas descritas, temos preferido utilizar a endoaneurismorrafia com prótese de Dacron, com fio de Polipropileno 3-0 em todas as anastomoses e, quando necessário, reforço com banda circular de Teflon ou Dacron.

Recentemente, KIRKLIN \& KOUCHOUKOS 13 estabeleceram os critérios de inclusāo do arco transverso no reparo das dissecçōes aórticas, com os quais concordamos, que sāo: 1) a presença da laceração intimal no arco; 2) rotura do arco; 3) fragilidade da parede externa da falsa luz e 4) fragmentação da parede interna da falsa luz.

Quando existe extensāo da dissecçāo ao arco aórtico, porém, a integridade anatômica está preservada; nós temos realizado a reconstrução parcial do mesmo ("hemiarch repair"). Entretanto, quando a laceração da íntima encontra-se dentro do arco ou se há rotura do mesmo, procedemos à reconstrução total do arco transverso e ao reimplante em bloco dos troncos supra-aórticos (Figura 2).

A perda de sustentação da válvula aórtica, ou mesmo a rotura de seus folhetos, ocorre em torno de $40 \%$ dos casos de dissecçōes do tipo A (em nossa série, $31,8 \%$ ) e deve ser corrigida mediante valvoplastia, se possível, ou com troca valvar. A despeito de alguns autores ${ }^{9}, 14$, que conseguem a 
ALBUQUERQUE, L. C.; GOLDANI, M. A.; GOLDANI, J. J.; PIANTÁ, R. M.; ARAÚJO, R. L.; PETRACCO, J. B. - Tratamento cirúrgico das dissecçōes de aorta tipo A utilizando parada cardiocirculatória total com hipotermia profunda. Rev. Bras. Cir. Cardiovasc., 8(1):1-8, 1993.

reconstrução da válva em um bom número de casos, nós conseguimos realizar esta técnica em apenas 2 pacientes, tenho sido necessária a troca valvar em 5 casos.

A mortalidade cirúrgica das dissecçōes do tipo A tem decrescido significativamente nos últimos anos, devido aos avanços já descritos. Em nossa série, a mortalidade hospitalar global de $18 \%$ é bastante aceitável em nosso meio e superponível à maioria das outras séries, como demonstrado no Quadro 1. O único fator preditivo de mortalidade maior foi a inclusāo do arco aórtico no reparo cirúrgico, reconhecidamente um fator de incremento do risco operatório.

Seguramente estes resultados satisfatórios devem-se à abordagem mais agressiva e, ao mesmo tempo, mais segura, proporcionada pelo uso da PCC total sob hipotermia profunda como rotina nas dissecçōes do tipo A, a qual associa eficácia de proteçăo cerebral à simplificação da correção cirúrgica.

\section{CONCLUSĀO}

A técnica de parada cardiocirculatória com hipotermia profunda é um método seguro de proteção cerebral que deve ser utilizado rotineiramente no reparo cirúrgico das dissecçōes aórticas do tipo $A$, pois: 1) permite melhor inspeção da extensão da zona dissecada e a determinação mais precisa do local de rotura da íntima, facilitando a decisão de incluir ou não o arco aórtico na reconstrução; 2) simplifica tecnicamente a correção, proporcionando um campo operatório adequado e melhor hemostasia $\mathrm{da}$ (s) anastomose(s) distal(is), e 3) impede as lesōes adicionais produzidas na parede da aorta, por abolir a necessidade do clampeamento de áreas freqüentemente englobadas pela dissecção. Esta técnica pode ser utilizada, inclusive, durante a anastomose proximal, em casos que não se extendam ao arco aórtico e que nāo apresentem insuficiência aórtica associada, sem riscos adicionais, desde que o tempo de PCC seja mantido abaixo de 60 minutos.

\section{RBCCV 44205-194}

ALBUQUerQUE, L. C.; GOLDANI, M. A.; GOLDANI, J. J.; PIANTÁ, R. M.; ARAÚJO, R. L.; PETRACCO, J. B. - Surgical treatment of type A aortic dissections utilizing total cardiocirculatory arrest with deep hypothermia. Rev. Bras. Cir. Cardiovasc., 8(1):1-8, 1993.

ABSTRACT: From July 1986 to July 1993, 22 consecutive patients with Type A Aortic Dissecation were surgically treated using total cardiovascular arrest with deep hypothermia $\left(18^{\circ} \mathrm{C}\right)$. Fifteen cases had acute dissecations and 7 were chronic cases. In 14 cases (64\%), the ascending aorta was reconstructed using a straight Dacron graft. In 5 of these, the aortic valve was repaired. The aortic arch was reconstructed in 8 cases $(36 \%)$ and in 3 of these, the arch branches were reimplanted. The average time of the cardiovascular arrest was 43 minutes and the hospital mortality was $18 \%$. The most frequent complication was respiratory infection, 5 patients $(22.7 \%)$ showed neurologic damage, 4 of them transient and reversible. In summary, the cardiovascular arrest with deep hypothermia technique for the treatment of Type A Aortic Dissection can be used with reasonable mortality and morbility allowing a safe cerebral protection, good exposure of the dissected aorta and prevents damage to the aortic wall by the cross clamping.

DESCRIPTORS: aorta, dissection, surgery; circulatory arrest; hypothermia, deep.

\section{REFERÊNCIAS BIBLIOGRÁFICAS}

1 BACHET, J.; GOUDOT, B.; TEODORI, G.; BRODATY, D.; DUBOIS, C.; LENTDECKER, D.; GUILMET, D. - Surgery of type A acute aortic dissection with gelatine-resorcine-formol biological glue: a twelveyear experience. J. Cardiovasc. Surg., 31: 263$273,1990$.

CABROL, C. E. A.; GANDJBAKHCH, I.; PAVIE, A. BORS, V.; JAULT, F. - Surgical treatment of aortic dissection at La Pitie Hospital. Semin. Thorac. Cardiovasc. Surg., 3: 245-250, 1991.

3 CARPENTIER, A. - "Glue aortoplasty" as an alternative to resection and grafting for the treatment of aortic dissection. Semin. Thorac. Cardiovasc. Surg., 3: 213-214, 1991.

4 COOLEY, D. A. - Experience with hypothermic circulatory arrest and the treatment of aneurysms of the ascending aorta. Semin. Thorac. Cardiovasc. 

$8(1): 1-8,1993$.

Surg., 3: 160-170, 1991.

COOLEY, D. A. - Surgical treatment of aortic aneurysms. Philadelphia, WB Saunders, 1986. p. 46-61.

6 CRAWFORD, E. S.; SVENSSON, L. G.; COSELLI, J. S.; SAFI, H. J.; HESS, K. R. - Surgical treatment of aneurysm and/or dissection of the ascending aorta, transverse aortic arch, and ascending aorta and transverse aortic arch: factors influencing survival in 717 patients. J. Thorac. Cardiovasc. Surg., 98: 659-674, 1989.

7 DAILY, P. O.; TRUEBLOOD, W.; STINSON, E. B.; WVERFLEIN, R. D.; SHUMWAY, N. E. - Management of acute aortic dissecations. Ann. Thorac. Surg., 10: 237-247, 1970.

DE BAKEY, M. E.; HENLEY, W. S.; COOLEY, D. A.; MORRIS, G. C.; CRAWFORD, E. S.; BEALL, A. C. - Surgical management of dissecting aneurysms of the aorta. J. Thorac. Cardiovasc. Surg., 49: 130140, 1965.

9 GALLOWAY, A. C.; COLVIN, S. B.; GROSSI, E. A.; PARISH, M. A.; CULLIFORD, A. T.; ASAI, T.; KOFSKY, N. M.; WEINREB, J. C.; SHAPIRO, S.; BAUMANN, F. G.; SPENCER, F. C. - Surgical repair of type $A$ aortic dissection by the circulatory arrestgraft inclusion technique in sixty-six patients. J. Thorac. Cardiovasc. Surg., 105: 781-790, 1993.
GRIEPP, E. B. \& GRIEPP, R. B. - Cerebral consequences of hypothermic circulatory arrest in adults. J. Cardiovasc. Surg., 7: 134-155, 1992.
11 HEINEMANN, M.; LASS, J.; JURMANN, M.; KARCK, M.; BORST, H. G. - Surgery extended into the aortic arch in acute type $A$ dissection. Circulation, 84 (Supl. 3): 25-30, 1991.

12 IKONOMIDIS, J. S.; WEISEL, R. D.; MOURADIAN, M. S.; IVANOV, J.; BAIRD, R. J.; MICKLEBOROUGH, L. L.; SCULLY, H. E.; GOLDMANS, B. S. - Thoracic aortic surgery. Circulation, 84 (Supl. 3): 1-6, 1991.

13 KIRKLIN, J. W. \& KOUCHOUKOS, N. T. - When and how to include arch repair in patients with acute dissections involving the ascending aorta. Semin. Thorac. Cardiovasc. Surg., 5: 27-32, 1993.

14 MILLER, D. C. - Surgical management of acute aortic dissection: new data. Semin. Thorac. Cardiovasc. Surg., 3: 225-237, 1991.

15 TABAYASHI, K.; NIIBORI, K.; IGUCHI, A.; SHOUJI, Y.; OHMI, M. MOHRI, H. - Replacement of the trasverse arch for type A aortic dissection. Ann. Thorac. Surg., 55: 864-867, 1993.

16 TAKAMOTO, S.; MATSUDA, T.; HADARA, M. - Simple hypothermic retrograde cerebral perfusion during aortic arch replacement: a preliminary report on two successful cases. J. Thorac. Cardiovasc. Surg., 104: 1106-1109, 1992.

17 VEDA, Y.; MIKI, S.; KUSUHARA, K. - Surgical treatment of the aneurysms or dissection involving the ascending aorta and aortic arch utilizing circulatory arrest and retrograde perfusion. J. Thorac. Surg., 36: $161-166,1988$. 